\title{
PRIMITIVE BLOCK DESIGNS WITH AUTOMORPHISM GROUP $\operatorname{PSL}(2, q)$
}

\author{
Snježana Braić, Joško Mandić and Tanja Vučičić \\ University of Split, Croatia
}

\begin{abstract}
We present the results of a research which aims to determine, up to isomorphism and complementation, all primitive block designs with the projective line $F_{q} \cup\{\infty\}$ as the set of points and PSL $(2, q)$ as an automorphism group. The obtained designs are classified by the type of a block stabilizer. The results are complete, except for the designs with block stabilizers in the fifth Aschbacher's class. In particular, the problem is solved if $q$ is a prime. We include formulas for the number of such designs with $q=p^{2^{\alpha} 3^{\beta}}, \alpha, \beta$ nonnegative integers.
\end{abstract}

\section{INTRODUCTION AND PRELIMINARIES}

Our aim is to determine, up to isomorphism and complementation, all nontrivial primitive block designs on the projective line with PSL $(2, q)$ as an automorphism group. For each $q$ we denote by $\operatorname{npd}(q)$ the number of such designs. We also determine which of the occurring 2-designs is even a 3 -design.

Several authors have considered the action of group PSL $(2, q)$ on the projective line. For this research the most significant contribution is the work of Cameron et al. [5,6], which we use in the part involving 3-designs with $q$ odd. Focusing on primitive designs only, we extend the results taken from $[5,6]$ by solving the problem of isomorphism of the designs and by finding their full automorphism groups. Additionally, our method yields the series of 3-designs (Proposition 5.2) undetected in $[5,6]$. The rest of the research comprises 2-designs and 3-designs with $q$ even.

The obtained designs are presented following the type of a block stabilizer. We completely solved the problem in case when a block stabilizer is not in the fifth Aschbacher's class and, in particular, for $q$ a prime number. In

2010 Mathematics Subject Classification. 05B05.

Key words and phrases. Block design, automorphism group, primitive action. 
Section 5 a part of the designs is described by an explicit base block and an automorphism group. In Section 6 we give the data determining the full automorphism groups for the rest of the designs. Section 7 is a contribution to the calculation of the numbers $\operatorname{npd}(q)$. Formulas for $\operatorname{npd}(q)$ are determined in case $q=p^{2^{\alpha} 3^{\beta}}, \alpha, \beta$ nonnegative integers. For instance, Proposition 7.1 relates to the case $\alpha=\beta=0$. The validity of the obtained formulas is illustrated through computer construction of designs up to $q=103$, for which we used software packages GAP $[9,15]$ and MAGMA [3] and the libraries of primitive groups that they contain.

We start with a few basic notions and facts that are relevant for our study. More details on design theory the reader can find, for instance, in [2,7], while for group theory we refer the reader to $[1,4,8]$. Our notation and terminology is in accordance with the cited literature.

A $t-(v, k, \lambda)$ design is a pair $D=(\Omega, \mathcal{B})$, where $\Omega$ is a set of $v$ points, $\mathcal{B}$ a set of $k$-sets of $\Omega$ called blocks, such that any $t$ different points are contained in exactly $\lambda$ blocks, $t \leq k$ and $\lambda>0$. Any $2-(v, k, \lambda)$ design we simply call $(v, k, \lambda)$ block design.

An isomorphism of $t$-designs $D=(\Omega, \mathcal{B})$ and $D^{\prime}=\left(\Omega, \mathcal{B}^{\prime}\right)$ is a permutation of $\Omega$ which sends blocks of $D$ to blocks of $D^{\prime}$. An isomorphism from $D$ to itself is called automorphism. The group of all automorphisms of $D$ is denoted by $\operatorname{Aut} D$. For any $\omega \in \Omega$ by $G_{\omega} \leq \operatorname{Aut} D$ we denote a point stabilizer; $G_{B} \leq$ Aut $D$ denotes a block stabilizer, $B \in \mathcal{B}$.

A permutation group $G$ acting transitively on a set $X,|X| \geq 2$, is primitive if each point stabilizer $G_{x}, x \in X$, is a maximal subgroup of $G$ $[8$, p. 14]. We call a $t-$ design $D$ primitive if there exists an automorphism group $G \leq \operatorname{Aut} D$ which acts primitively on the point and block sets.

It is known that 2-homogeneous permutation groups are primitive $[8$, p. 35], thus all 2-transitive permutation groups are primitive.

Proposition 1.1 ([8, p. 9]). Let $G$ be a permutation group acting transitively on a set $X$. Then a subgroup $L \leq G$ is transitive if and only if $G=L G_{x}$, $x \in X$.

An overgroup of a primitive group is primitive. All primitive groups that have the same socle with a specified (transitive) permutation action form a cohort [8, p.138]. The primitive groups $G$ of this research are almost simple, i.e. $T \triangleleft G \leq$ Aut $T, T$ nonabelian simple, [13]. The definition of Aschbacher's classes can be found in [11].

We denote by $F_{q}$ a finite field with $q$ elements; we also set $q=p^{f}, p$ a prime, $F_{q}^{*}=F_{q} \backslash\{0\}$ and $F_{q}^{(2)}=\left\{x^{2} \mid x \in F_{q}^{*}\right\}$. We consider primitive designs with respect to $G$, such that

$$
\operatorname{PSL}(2, q)=T \unlhd G \leq \operatorname{Aut} T=P \Gamma L(2, q) .
$$


The socle $T$ of the cohort (1.1) is the group of all fractional linear transformations

$$
t_{a, b, c, d}: z \mapsto \frac{a z+b}{c z+d}
$$

on the projective line $F_{q} \cup\{\infty\}, a, b, c, d \in F_{q}$, where $a d-b c$ is a square; $|T|=\frac{q\left(q^{2}-1\right)}{\operatorname{gcd}(2, q-1)}$.

Let $\xi$ be a primitive element of $F_{q}$ and let $\delta=t_{\xi, 0,0,1}$. By $\phi$ we denote the automorphism $z \mapsto z^{p}$ of $F_{q}$ which fixes $\infty$. Then we have

$$
\begin{aligned}
& \operatorname{PGL}(2, q)=\langle\operatorname{PSL}(2, q), \delta\rangle=\langle T, \delta\rangle, \quad|\operatorname{PGL}(2, q)|=q\left(q^{2}-1\right) ; \\
& P \Gamma L(2, q)=\langle\operatorname{PSL}(2, q), \delta, \phi\rangle=\langle T, \delta, \phi\rangle, \quad|P \Gamma L(2, q)|=f q\left(q^{2}-1\right) ; \\
& P \Sigma L(2, q)=\langle\operatorname{PSL}(2, q), \phi\rangle=\langle T, \phi\rangle ; \quad|P \Sigma L(2, q)|=\frac{f q\left(q^{2}-1\right)}{\operatorname{gcd}(2, q-1)} .
\end{aligned}
$$

Our theoretical considerations are restricted to $q \geq 13, q \neq 23$.

\section{Construction method}

The basis of our construction method is the following theorem.

THEOREM 2.1. [2, p.175] Let $G$ be a permutation group on the finite set $\Omega$, let $B \subset \Omega$ be a k-subset with at least two elements and let $G_{B} \leq G$ be a setwise stabilizer of $B$.

If $G$ is t-homogeneous and $k \geq t$, then $D=\left(\Omega, B^{G}=\left\{B^{\gamma} \mid \gamma \in G\right\}\right)$ is a t-design with $b=\left|B^{G}\right|=|G| /\left|G_{B}\right|$ blocks and

$$
\lambda=b\left(\begin{array}{l}
k \\
t
\end{array}\right) /\left(\begin{array}{l}
v \\
t
\end{array}\right)=|G|\left(\begin{array}{l}
k \\
t
\end{array}\right) /\left|G_{B}\right|\left(\begin{array}{l}
v \\
t
\end{array}\right) .
$$

The set $B$ is called a base block for $D$.

We take $\Omega=F_{q} \cup\{\infty\},|\Omega|=q+1$. It is well-known that $T$-action on projective line is 2 -homogeneous if $q \equiv 1(\bmod 4)$, whereas it is 3 -homogeneous if $q \equiv 3(\bmod 4)$ or $q$ is even. It is also known that $\operatorname{PGL}(2, q)$ acts 3 homogeneously on projective line for all $q$. If, for a given group $G$ from (1.1), we select a subset $B \subset \Omega$ and construct the set $\mathcal{B}=B^{G}$, then the pair $D=(\Omega, \mathcal{B})$ is a 2 or 3 -design with a base block $B$ and $G \leq \operatorname{Aut} D$ (Theorem 2.1). $G$ obviously acts primitively on the set of points of $D$. If the base block stabilizer $G_{B} \leq G$ is a maximal subgroup, then $G$ acts primitively on blocks as well. Consequently, in order to construct a primitive design $D$ in this way, it suffices to choose $B$ to be a union of orbits of some maximal subgroup of $G$. Hence we denote by $D(G, B)$ such a design. We only consider possible nontrivial choices for $B$ with the property $k=|B| \leq v / 2$ because the complement of a primitive design is also primitive with the same full automorphism group. If the action of $\operatorname{Aut} D(G, B)$ is 3-homogeneous then the underlying design is a 3-design. 


\section{Minimal group for Performing the COnStruction}

Suppose that for primitive design $D(G, B)$ we have $T \leq G_{1} \leq G$. Then $G_{1}$ is normal subgroup of $G$, so $G_{1}$ acts transitively on blocks. Now, if $G_{B} \cap G_{1}$ is maximal in $G_{1}$, then the constructions by $G_{1}$ produce also all primitive designs admitting $G$ as an automorphism group. The consequence of this simple observation is that, eventually, we only need to consider maximal subgroups of $T$ and $\operatorname{PGL}(2, q)$ as setwise base block stabilizers to construct all desired primitive designs. Namely, if $G$ is any group from (1.1) and $M \leq G$ its maximal subgroup not contained in $T$, then $M \cap T$ is maximal in $T$ with one single exception: normalizer $M=N_{G}\left(A_{4}\right)=S_{4}$ in $G=\operatorname{PGL}(2, q)$ and $q=p \equiv \pm 11,19(\bmod 40)$ [10, Theorem 1.1 and Corollary 1.2$]$.

\begin{tabular}{|c|c|c|c|c|c|}
\hline Type & $H \leq T$ (block stabilizer) & Asch. & $G^{M I N}$ & $n c c$ & $G^{M A X}$ \\
\hline 1 & $\begin{array}{l}C_{p}^{f} \rtimes C_{\frac{q-1}{\operatorname{gcd}(2, q-1)}} \\
\text { (point stabilizer) }\end{array}$ & $C_{1}$ & $\operatorname{PSL}(2, q)$ & 1 & $P \Gamma L(2, q)$ \\
\hline 2 & $\begin{array}{l}D_{\frac{2(q-1)}{\operatorname{gcd}(2, q-1)}} \\
\left(\begin{array}{l}\text { two points } \\
\text { setwise stabilizer }\end{array}\right)\end{array}$ & $C_{2}$ & $\operatorname{PSL}(2, q)$ & 1 & $P \Gamma L(2, q)$ \\
\hline 3 & $D_{\frac{2(q+1)}{\operatorname{gcd}(2, q-1)}}$ & $C_{3}$ & $\operatorname{PSL}(2, q)$ & 1 & $P \Gamma L(2, q)$ \\
\hline 4 & $\operatorname{PGL}\left(2, q_{0}\right), q=q_{0}^{2}$ & $C_{5}$ & $\operatorname{PSL}(2, q)$ & $2^{1}$ & $P \Sigma L(2, q)$ \\
\hline 5 & $\begin{array}{l}\operatorname{PSL}\left(2, q_{0}\right), q=q_{0}^{r} \\
q_{0} \neq 2, r \text { odd prime }\end{array}$ & $C_{5}$ & $\operatorname{PSL}(2, q)$ & 1 & $P \Gamma L(2, q)$ \\
\hline 6 & $\begin{array}{l}A_{5}, q=p^{2} \equiv 49(\bmod 60) \\
p \equiv 7,13,17,23,37,43 \\
47,53(\bmod 60)\end{array}$ & $C_{9}$ & $\operatorname{PSL}(2, q)$ & 2 & $P \Sigma L(2, q)$ \\
\hline 7 & $\begin{array}{l}A_{5}, q=p \equiv 1,11,19 \\
29,31,41,49,59(\bmod 60)\end{array}$ & $C_{9}$ & $\operatorname{PSL}(2, q)$ & 2 & $\operatorname{PSL}(2, q)$ \\
\hline 8 & $\begin{array}{l}A_{4}, q=p \equiv 13,37,43,53 \\
67,77,83,107(\bmod 120)\end{array}$ & $C_{6}$ & $\operatorname{PSL}(2, q)$ & 1 & $\operatorname{PGL}(2, q)$ \\
\hline 9 & $\begin{array}{l}S_{4}, q=p \equiv 1,7,17,23,31 \\
41,47,49,71,73,79,89 \\
97,103,113,119(\bmod 120)\end{array}$ & $C_{6}$ & $\operatorname{PSL}(2, q)$ & 2 & $\operatorname{PSL}(2, q)$ \\
\hline 10 & $\begin{array}{l}S_{4}, q=p \equiv 11,19,29,59 \\
61,91,101,109(\bmod 120)\end{array}$ & $C_{6}$ & $\operatorname{PGL}(2, q)$ & 1 & $\operatorname{PGL}(2, q)$ \\
\hline
\end{tabular}

TABLE 1 
Maximal subgroups of $T$ can, for instance, be read off from [10, Theorem 2.1 and Theorem 2.2]. Here we rewrite that division into nine isomorphism types following the orbit structure. The types are listed in the first nine rows of Table 1 and denoted by $H$. The corresponding Aschbacher's class ([11]) is indicated in the third column.

In Table $1 G^{M I N}$ denotes minimal groups from (1.1) with maximal subgroup $H$, i.e. minimal primitive automorphism groups of the prospective designs. The tenth row of Table 1 relates to the case $G^{M I N}=\operatorname{PGL}(2, q)$; here the socle $T$ of cohort (1.1) does not act primitively on blocks.

Group $G^{M A X}$ will be defined and explained in the next section. The fifth column reads the number $n c c$ of conjugacy classes of $H$ in $G^{M I N} ; n c c \leq 2$ for any isomorphism type of maximal subgroup of $T$.

To accomplish the construction of all our aimed designs it suffices to:

(i) Compose base blocks as all possible unions of $H$-orbits, $H$ of type 2 thru 10. (Block stabilizer $H$ of type 1 has two orbits whose lengths are 1 and $q$. The corresponding design is obviously trivial.)

(ii) Generate the block set $\mathcal{B}$ from each base block $B$ by the action of

$$
\left\{\begin{array}{l}
T \text { on } B, \quad \text { for } H \text { of type } 2 \text { to } 9 \\
\operatorname{PGL}(2, q) \text { on } B, \quad \text { for } H \text { of type } 10 .
\end{array}\right.
$$

\section{Preliminary analysis of Designs}

In this section we give important facts about full automorphism groups and possible isomorphisms of the designs we consider. By $H$ we denote a maximal subgroup of $T$.

The following assertion has been observed in [5, Introduction] and [12].

Proposition 4.1. Let $G$ belong to the cohort (1.1) and let $D=D(G, B)$ and $D^{\prime}=D\left(G, B^{\prime}\right)$ be any two considered designs. If $\pi: D \rightarrow D^{\prime}$ is an isomorphism, then $\pi \in P \Gamma L(2, q)$.

Taking $D^{\prime}=D$, from the proposition it follows that each considered design $D$ has the property Aut $D \leq P \Gamma L(2, q)$. Isomorphisms from $P \Gamma L(2, q)$ act on the set of blocks of a design in the sense that they preserve $T$ as the generating group:

$$
\left(B^{T}\right)^{\pi}=B^{T \pi}=B^{\pi \pi^{-1} T \pi}=\left(B^{\pi}\right)^{T}, \pi \in P \Gamma L(2, q) .
$$

For a given design $D=D(T, B)$, the set $\operatorname{stb}(D)=\left\{T_{B^{*}} \mid B^{*} \in \mathcal{B}=B^{T}\right\}$ of stabilizers of all blocks of $D$ we call $D$-stabilizer. It is the set of all subgroups conjugate to some block stabilizer $H=T_{B}$, i.e. $\operatorname{stb}(D)=H^{T}$. For an isomorphism $\pi: D \rightarrow D^{\prime}$ we have $(s t b(D))^{\pi}=s t b\left(D^{\prime}\right)$, cf. (4.1). This means that we obtain all designs up to isomorphism if the construction (i)-(ii) is performed only for a chosen representative of every conjugacy class of $H$ in $P \Gamma L(2, q)$. Clearly, $P \Gamma L(2, q)$ acts on the classes of T. So if $H=T_{B}$ for the 
design $D(G, B)$ then by $G^{M A X}$ we denote the set stabilizer of the conjugacy class of $H$ in $T$. Obviously, Aut $D \leq G^{M A X}$ and

$$
G^{M A X}=\left\{\begin{array}{l}
P \Gamma L(2, q), \text { if there exists one conjugacy class of } H \text { in } T ; \\
P \Sigma L(2, q), \text { if } q \text { is odd and there exist two conjugacy } \\
\text { classes of } H \text { in } T .
\end{array}\right.
$$

From Proposition 1.1 we deduce

$$
G^{M A X}=T \cdot N_{G^{M A X}}(H) .
$$

$N_{G^{M A X}}(H)$ acts on the set of $H$-orbits and, accordingly, on the block set of the design.

Proposition 4.2. Let $D_{1}=D\left(T, B_{1}\right)$ and $D_{2}=D\left(T, B_{2}\right)$ be designs with $T_{B_{1}}=T_{B_{2}}=H$. Then $D_{1}$ and $D_{2}$ are isomorphic if and only if there exists $\pi \in N_{G^{M A X}}(H)$ so that $B_{1}^{\pi}=B_{2}$.

Proof. Let $\varphi: D_{1} \rightarrow D_{2}$ be an isomorphism. Then there exists $g \in T$ such that $B_{1}^{\varphi}=B_{2}^{g}$, and for the isomorphism $\pi=\varphi g^{-1}: D_{1} \rightarrow D_{2}$ we have $B_{1}^{\pi}=B_{2}$. Now $H=T_{B_{2}}=T_{B_{1}^{\pi}}=T_{B_{1}}^{\pi}=H^{\pi}$, which proves $\pi \in N_{G^{M A X}}(H)$.

Conversely, let there exist $\pi \in N_{G^{M A X}}(H)$ such that $B_{1}^{\pi}=B_{2}$. Then

$$
B_{2}^{T}=\left(B_{1}^{\pi}\right)^{T} \stackrel{(4.1)}{=}\left(B_{1}^{T}\right)^{\pi}
$$

i.e. $\pi$ is an isomorphism.

Corollary 4.3. Aut $D=T \cdot\left\{\pi \in N_{G^{M A X}}(H) \mid B^{\pi}=B\right\}$.

Proof. Proposition 1.1 implies Aut $D=T \cdot \operatorname{Aut} D_{B}$, while previous proposition with $D_{1}=D_{2}=D$ gives Aut $D_{B}=\left\{\pi \in N_{G^{M A X}}(H) \mid B^{\pi}=B\right\}$.

\section{Designs With BlOCK StABilizers OF types 2 Thru 5}

In this and the subsequent section we describe all primitive 2-designs $D$ with $\operatorname{PSL}(2, q) \unlhd \operatorname{Aut} D$ on $q+1$ points up to one undecided case. For the description and for solving the problem of possible isomorphism between two designs we use group $N_{G^{M A X}}(H)$. Henceforth that group we denote by $K$.

For $H$-types 2 thru 5 we explicitly give $H$-orbits and a base block of the design. The description is incomplete for $H$-type 5 in the sense that we found orbit lengths for $H$ but not for $K$.

A) $H$-type 2 (a dihedral group, two point stabilizer)

Proposition 5.1. Let $q \geq 13$. A block design $D$ with the socle PSL $(2, q)$ of Aut $D$ and the base block stabilizer $H$ in the second Aschbacher's class exists if and only if $q \equiv 1(\bmod 4)$. Then $D$ is $2-\left(q+1, \frac{q-1}{2}, \frac{(q-1)(q-3)}{8}\right)$ design which is unique up to isomorphism and complementation. Moreover, Aut $D=$ $P \Sigma L(2, q)$. 
Proof. If $D$ exists then

$$
H=T_{\{0, \infty\}}=\left\{x \mapsto a x: a \in F_{q}^{(2)}\right\} \rtimes\left\langle x \mapsto \frac{-1}{x}\right\rangle,
$$

$G^{M A X}=P \Gamma L(2, q)$ and $K=P \Gamma L(2, q)_{\{0, \infty\}} \cdot$

The orbits of subgroup $\left\{x \mapsto a x \mid a \in F_{q}^{(2)}\right\}$ are $\{\infty\},\{0\}, F_{q}^{(2)}$ and $F_{q}^{*} \backslash F_{q}^{(2)}$. Consequently,

$$
H-\text { orbits are }\left\{\begin{array}{l}
\{0, \infty\} \text { and } F_{q}^{*}, \quad \text { for } q \text { even or } q \equiv 3(\bmod 4) ; \\
\{0, \infty\}, F_{q}^{(2)} \text { and } F_{q}^{*} \backslash F_{q}^{(2)}, \quad \text { for } q \equiv 1(\bmod 4) .
\end{array}\right.
$$

Thus, nontrivial 2-designs exist for $q \equiv 1(\bmod 4), q \geq 13$. Up to complementation it remains to consider base blocks consisting of one orbit each, that being $B_{1}=F_{q}^{(2)}$ and $B_{2}=F_{q}^{*} \backslash F_{q}^{(2)}$.

Obviously the mapping $x \mapsto \xi x, \xi \in F_{q}^{*} \backslash F_{q}^{(2)}$, lies in $K$ and maps the orbit $F_{q}^{(2)}$ into $F_{q}^{*} \backslash F_{q}^{(2)}$, so up to isomorphism and complementation there exists a unique $2-\left(q+1, \frac{q-1}{2}, \frac{(q-1)(q-3)}{8}\right)$ design $D=D\left(T, F_{q}^{(2)}\right)$. Using Corollary 4.3 we easily get Aut $D=P \Sigma L(2, q)$.

B) $H$-type 3 (a dihedral group of order $\left.\frac{2(q+1)}{\operatorname{gcd}(2, q-1)}\right)$

Proposition 5.2. Let $q=p^{f} \geq 13$. A block design $D$ with the socle PSL $(2, q)$ of Aut $D$ and the base block stabilizer $H$ in the third Aschbacher's class exists if and only if $q \equiv 1(\bmod 4) . D$ is unique up to isomorphism and complementation. If $p \equiv 1(\bmod 4)$, then $D$ is a 2 $\left(q+1, \frac{q+1}{2}, \frac{(q-1)^{2}}{8}\right)$ design with Aut $D=P \Sigma L(2, q)$. If $p \equiv 3(\bmod 4)$, then $D$ is a $3-\left(q+1, \frac{q+1}{2}, \frac{(q-3)(q-1)}{16}\right)$ design with Aut $D=\operatorname{PSL}(2, q) \cdot \Delta$, where $\Delta$ is cyclic group of order $2 f$ and $|\operatorname{PSL}(2, q) \cap \Delta|=2$.

Proof. If $q \equiv 3(\bmod 4)$ or $q$ is even, then $H$ acts transitively on the projective line, which leaves the possibility $q \equiv 1(\bmod 4)$, so $H$ is a dihedral group of order $q+1$ and $G^{M A X}=P \Gamma L(2, q)$. In [6, Lemma 14, (i)] we find that $H$ acts in two orbits on $\Omega$.

Let $F_{q}^{*}=\langle\xi\rangle$ and $A=\left\{x \mapsto \frac{a x+b \xi}{b x+a}: a, b \in F_{q}, a^{2}-b^{2} \xi \in F_{q}^{(2)}\right\}$. Then $A$ is a cyclic subgroup of $H$ of order $\frac{q+1}{2}$ and $H=N_{T}(A)$. One $H$-orbit on $\Omega$ is $\{\infty\} \cup\left\{a \in F_{q}^{*}: a^{2} \in \xi+F_{q}^{(2)}\right\}$. Up to isomorphism and complementation, design $D=D\left(T,\{\infty\} \cup\left\{a \in F_{q}^{*}: a^{2} \in \xi+F_{q}^{(2)}\right\}\right)$ is a unique $2-\left(q+1, \frac{q+1}{2}, \frac{(q-1)^{2}}{8}\right)$ design with the base block stabilizer $A \rtimes \Delta$, where $\Delta=\left\{\delta_{u} \mid u \in \mathbb{Z}\right\}$ is a cyclic group of order $2 f$ and $\delta_{u}$ is the action on $\Omega$ defined 
by $x \rightarrow \xi^{\frac{1-p^{u}}{2}} x^{p^{u}}\left(\delta_{u_{1}} \circ \delta_{u_{2}}=\delta_{u_{1}+u_{2}}\right)$. Corollary 4.3 implies Aut $D=T \cdot \Delta$. If $p \equiv 3(\bmod 4)$, then $\xi^{\frac{1-p}{2}}$ is not a square, so Aut $D$ is 3 -homogeneous.

REMARK 5.3. 3-designs from the above proposition are not given in $[5,6]$. The group PSL $(2, q) \cdot \Delta$ has the same order as $P \Sigma L(2, q)$ but is different from this group.

C) $H$-type $4\left(H \cong \operatorname{PGL}\left(2, q_{0}\right), q=q_{0}^{2}\right)$

Proposition 5.4. Let $q=q_{0}^{2} \geq 13$. Then, up to isomorphism and complementation, there exists a unique primitive block design $D$ with automorphism group PSL $(2, q)$ and a block stabilizer $H=\operatorname{PGL}\left(2, q_{0}\right)$. Aut $D=P \Sigma L(2, q)$ and one of the following holds:

(1) $q$ is even and $D$ is a $3-\left(q_{0}^{2}+1, q_{0}+1,1\right)$ design;

(2) $q$ is odd and $D$ is a $2-\left(q_{0}^{2}+1, q_{0}+1, \frac{q_{0}+1}{2}\right)$ design.

Proof. If $q$ is odd, in [6, Lemma 14, (i)] we find that $H$ acts in two orbits on $\Omega$. If $q$ is even, then $H$ consists of all fractional linear transformations in $T$ with coefficients from $F_{q_{0}}$. Obviously, $\{\infty\} \cup F_{q_{0}}$ is a $H$-orbit. Let $\gamma \in F_{q} \backslash F_{q_{0}}$. $\gamma$ generates $F_{q}$ and every element in $F_{q} \backslash F_{q_{0}}$ can be presented in the form $a \gamma+b, a \in F_{q_{0}}^{*}, b \in F_{q_{0}} . H$ contains the group $\left\{x \mapsto a x+b: a \in F_{q_{0}}^{*}, b \in F_{q_{0}}\right\}$, so $F_{q} \backslash F_{q_{0}}$ is also a $H$-orbit, i.e. $H$ acts in two orbits on $\Omega$ also for $q$ even. Thus, because $D\left(T, F_{q} \backslash F_{q_{0}}\right)$ is complementary to $D=D\left(T, F_{q_{0}} \cup\{\infty\}\right), D$ is a unique existing $2-\left(q_{0}^{2}+1, q_{0}+1, \frac{q_{0}+1}{\operatorname{gcd}\left(2, q_{0}-1\right)}\right)$ design up to isomorphism and complementation; Aut $D=P \Sigma L(2, q)$. For $q$ even, the group $P \Sigma L(2, q)$ is 3 -homogeneous, so $D$ is a $3-\left(q_{0}^{2}+1, q_{0}+1,1\right)$ design.

Designs from the above proposition, (1), are called Möbius planes ([7, p. $82])$.

D) $H$-type $5\left(H \cong \operatorname{PSL}\left(2, q_{0}\right), q=q_{0}^{r}, q_{0} \neq 2, r>2\right.$ prime)

For this $H$-type we only partly solved the problem by finding orbit structure for $H$. Orbit structure for $K=N_{P \Gamma L(2, q)}(H)$ remained beyond our reach because of the great number of combinatorial possibilities for the action of the automorphisms of $F_{q}$ (contained in $K$ ) on $H$-orbits.

If $q$ is odd, from [6, Lemma 14, (ii)] it follows that $\{\infty\} \cup F_{q_{0}}$ is the only $H$-orbit which is not regular. In case $q$ is even, $H$ consists of all elements in $T$ with coefficients $a, b, c, d \in F_{q_{0}}, q_{0} \neq 2$ is a prime power. Obviously, $\{\infty\} \cup F_{q_{0}}$ is a $H$-orbit. Let $\gamma \in F_{q} \backslash F_{q_{0}}$. Because $r$ is prime, $\gamma$ generates $F_{q}$. Let $x \mapsto \frac{a x+b}{c x+d}$ be an element of $H$ which stabilizes $\gamma$. Then $\frac{a \gamma+b}{c \gamma+d}=\gamma$, i.e. $c \gamma^{2}+(d-a) \gamma-b=0 . c \neq 0$ would imply that $\gamma$ is a root of a polynomial of degree 2 with coefficients in $F_{q_{0}}$, which is a contradiction. Thus $c=0, a=d$ and $b=0$, which means that points in $F_{q} \backslash F_{q_{0}}$ have trivial stabilizer and that $\{\infty\} \cup F_{q_{0}}$ is the only non regular H-orbit also in case $q$ is even. 
Consequently, for $H$-orbit lengths we find $\left(q_{0}+1\right)^{1}\left|\operatorname{PSL}\left(2, q_{0}\right)\right|^{s_{r}}$, where $s_{r}=\frac{q_{0}^{r-1}-1}{q_{0}^{2}-1} \cdot \operatorname{gcd}\left(2, q_{0}-1\right)$. Substituting $q_{0}=p^{f / r}$ we can write $s_{r}=$ $\frac{p^{f(1-1 / r)}-1}{p^{2 f / r}-1} \cdot \operatorname{gcd}(2, p-1)$ as well.

Design $D=D\left(T,\{\infty\} \cup F_{q_{0}}\right)$ is $3-\left(q_{0}^{r}+1, q_{0}+1,1\right)$ design called spherical geometry, [7, p. 82]; Aut $D=P \Gamma L(2, q)$.

In case $r=3$ we can easily describe all existing designs because $s_{3} \in$ $\{1,2\}$. If $q$ is even then $s_{3}=1$, so there exist only spherical geometry and its complement. If $q$ is odd then $s_{3}=2$. Let $\gamma \in F_{q} \backslash F_{q_{0}}$ generate $F_{q}$. There exists $\pi \in F_{q_{0}}^{*}$ such that $\pi \notin F_{q}^{(2)}$. Now $\gamma$ and $\pi \gamma$ lie in different orbits as an equation $\frac{a \gamma+b}{c \gamma+d}=\pi \gamma$ with $a, b, c, d \in F_{q_{0}}$ is impossible. In this case there exists exactly one more design (up to isomorphism and complementation), that being $D^{+}=$ $D\left(T, \gamma^{\mathrm{PSL}\left(2, q_{0}\right)}\right) . D^{+}$is $2-\left(q_{0}^{3}+1, \frac{q_{0}\left(q_{0}^{2}-1\right)}{2}, \frac{\left(q_{0}^{3}-1\right)\left(q_{0}\left(q_{0}^{2}-1\right)-2\right)}{4}\right)$ design. If $q \equiv$ $3(\bmod 4)$, then $D^{+}$is $3-\left(q_{0}^{3}+1, \frac{q_{0}\left(q_{0}^{2}-1\right)}{2}, \frac{\left(q_{0}\left(q_{0}^{2}-1\right)-2\right)\left(q_{0}\left(q_{0}^{2}-1\right)-4\right)}{8}\right)$ design.

\section{On the Designs obtained for $H$-Types 6 Thru 10}

In this section we consider designs with block stabilizers $H$ from the last five rows of Table 1, i.e. $H \cong A_{4}, S_{4}, A_{5} \leq \operatorname{PSL}(2, q)$ and $H \cong S_{4} \leq \operatorname{PGL}(2, q)$. We determine the number of designs and their full automorphism groups using orbit lengths of groups $H$ and $K$; here either $K=H$ or $[K: H]=2$. Orbit lengths for groups $H$ and $K$, in case of $H$-types 7-10, can be found in [5,6], as well as $H$-orbit lengths in case of $H$-type $6\left(q=p^{2}\right)$. On the other hand, if $H$ is of type 6 then $K$-orbit sizes can not be read off from the papers of Cameron et al. Therefore, subsequently in the section, we give in detail only the determining of $K$-orbit lengths for $H$-type 6 .

Let us begin with the calculation of the numbers $\operatorname{npd}_{H}(q)$ of nontrivial primitive $t$-designs having a particular block stabilizer $H$, regarded up to isomorphism and complementation. Let $\theta$ be the number of $H$-orbits. If $K=H$, obviously $\operatorname{npd}_{H}(q)=\left(2^{\theta}-2\right) / 2=2^{\theta-1}-1$. In case $[K: H]=2$ let $l \geq 0$ be the number of $H$-orbits that $K$ fixes setwise. Then $\theta-l$ is even, say $\theta-l=2 j, j \geq 1$. Let $\mathcal{O}=\left\{o_{11}, o_{21}, o_{12}, o_{22}, \ldots, o_{1 j}, o_{2 j}\right\}$ be the set of $H$ orbits that $K$ does not fix setwise, where $\left\{o_{1 i}, o_{2 i}\right\}, i=1, \ldots, j$ are $K$-orbits on $\mathcal{O}$. If we denote by $\Lambda$ the number of nonisomorphic designs with base blocks $\widetilde{B} \subseteq \mathcal{O}$, then we have $\operatorname{npd}_{H}(q)=\left[2^{l} \cdot \Lambda-2\right] / 2$. In order to calculate $\Lambda$ one can observe $2 \times j$ matrices $A=\left[A_{m i}\right]$ whose 0,1 entries correspond to the specific base block $\widetilde{B}$ in the sense that

$$
A_{m i}=\left\{\begin{array}{l}
1, o_{m i} \subseteq \widetilde{B} \\
0, o_{m i} \nsubseteq \subseteq \widetilde{B}
\end{array} .\right.
$$


The action of $K \backslash H$ on $\mathcal{O}$ and the consequent development of $\widetilde{B}$ reflect in the entries of $A$ as swapping the position of the rows of $A$. Eventually, we use the following lemma to determine $\Lambda$.

Lemma 6.1. Let $\mathcal{A}$ be the set of all $2 \times j$ matrices with 0,1 entries. For $A_{1}, A_{2} \in \mathcal{A}$ we define $A_{1} \sim A_{2}$ if and only if $A_{1}=A_{2}$ or $A_{2}$ is obtained from $A_{1}$ by swapping the rows. Then $\sim$ is an equivalence relation and $|\mathcal{A} / \sim|=$ $2^{2 j-1}+2^{j-1}$.

Proof. Obviously $|\mathcal{A}|=2^{2 j}$. It is easily checked that $\sim$ is an equivalence relation with 1 or 2 elements in each equivalence class. A class consists of only one matrix if the columns of that matrix are of the form $\left[\begin{array}{l}0 \\ 0\end{array}\right]$ or $\left[\begin{array}{l}1 \\ 1\end{array}\right]$, so the number of singleton classes is $2^{j}$. Let's denote the number of classes with two elements by $\mu$. Then we have $2^{j}+2 \mu=2^{2 j}$ or $\mu=2^{2 j-1}-2^{j-1}$. From $|\mathcal{A} / \sim|=2^{j}+\mu$ we finally obtain $|\mathcal{A} / \sim|=2^{2 j-1}+2^{j-1}$.

From the lemma we conclude that for a given block stabilizer $H$ with $[K: H]=2$ we have $\Lambda=2^{2 j-1}+2^{j-1}$ and consequently $\operatorname{npd}_{H}(q)=\left[2^{l}\right.$. $\left.\left(2^{2 j-1}+2^{j-1}\right)-2\right] / 2$.

E) $H$-type $6\left(H \cong A_{5}\right)$

Here $q=p^{2} \equiv 49(\bmod 60), p \equiv 7,13,17,23,37,43,47,53(\bmod 60),[10$, Theorem 2.2]. There are two conjugacy classes of $H$ in $T$, so $G^{M A X}=$ $P \Sigma L(2, q) ; K=S_{5}$. According to [6, Lemma 11, (i)] the only possible combination of $H$-orbit lengths is: $20^{1} 30^{1} 60^{\frac{q-49}{60}}$. $H$-orbits of length 20 and 30 are obviously fixed by $K$-action. On $K$-orbits of length 20, 30 and $60 H$ acts transitively, so for any point $\omega$ from these orbits $K_{\omega} \not \leq H$ holds. Let $\tau \in K \backslash H$ be an involution. All such involutions are conjugate in $K$, thus they fix the same number of points, say $\varphi$. The list of possibilities for $K$ action on the orbits of length $m$ is obtained by computer calculations ([9], $[3])$ :

\begin{tabular}{|l||l|l|l|l|l|}
\hline$m$ & 20 & 20 & 30 & 30 & 60 \\
\hline$K_{\omega}$ & $S_{3}$ & $C_{6}$ & $C_{4}$ & $C_{2}^{2}$ & $C_{2}$ \\
\hline$\varphi$ & 6 & 2 & 0 & 6 & 6 \\
\hline
\end{tabular}

If $K$ acts on an orbit of length 20, then $\tau$ has at least two fixed points in that orbit. Without loss of generality we may take that $\tau$ fixes points 0 and $\infty$; namely, such a choice of $K$ can be obtained by the action of some element from $G^{M A X}$. Then $x^{\tau}=a x^{p}$, where $a \in F_{q}^{(2)}$ and $a^{p+1}=1$. The equation $x^{p-1}=a^{-1}$ has $p-1$ solutions in $F_{q}^{*}$. These solutions are fixed points of $\tau$, so that $\tau$ has exactly $p+1$ fixed points. Let $\varphi_{1}, \varphi_{2}$ be the numbers of fixed points in orbits of length 20 and 30, respectively; $\varphi_{1} \in\{2,6\}, \varphi_{2} \in\{0,6\}$. Let $d$ be the number of orbits of length 60 fixed by involution $\tau$. Then the 
equation

$$
\varphi_{1}+\varphi_{2}+6 d=p+1
$$

holds. By substituting all admissible $\varphi_{1}$ and $\varphi_{2}$ into equation (6.1) and then solving it for $d$ we finally obtain orbit lengths for $K$ :

1. $p \equiv 13,37(\bmod 60) \rightarrow 20^{1} 30^{1} 60^{\frac{p-1}{6}} 120^{\frac{1}{2}\left(\frac{q-49}{60}-\frac{p-1}{6}\right)}$,

2. $p \equiv 17,53(\bmod 60) \rightarrow 20^{1} 30^{1} 60^{\frac{p-5}{6}} 120^{\frac{1}{2}\left(\frac{q-49}{60}-\frac{p-5}{6}\right)}$,

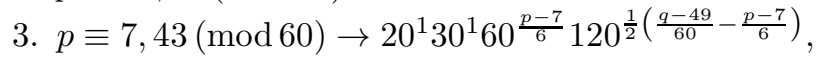

4. $p \equiv 23,47(\bmod 60) \rightarrow 20^{1} 30^{1} 60^{\frac{p-11}{6}} 120^{\frac{1}{2}\left(\frac{q-49}{60}-\frac{p-11}{6}\right)}$.

Obviously the following assertion holds.

Proposition 6.2. Let $p \geq 5$ and let $S_{5}$ be a maximal subgroup of $P \Sigma L\left(2, p^{2}\right)$. If orbit lengths of $S_{5}$ are $20^{1} 30^{1} 60^{d} 120^{d_{1}}, d_{1}=\frac{1}{2}\left(\frac{q-49}{60}-d\right)$, then for the number of primitive designs with $q=p^{2}$ we have the following:

1) If $p \not \equiv 49(\bmod 60)$, then $\operatorname{npd}(q)=3$.

2) If $p \equiv 49(\bmod 60)$, then $\operatorname{npd}(q)=2+2^{d+1}\left(2^{2 d_{1}-1}+2^{d_{1}-1}\right)$.

For $q=p^{2} \equiv 49(\bmod 60)$ we obtain the series of $2-\left(q+1, k, \frac{(q-1) k(k-1)}{120}\right)$ designs $D$ with $\operatorname{PSL}(2, q) \leq \operatorname{Aut} D \leq P \Sigma L(2, q)$.

For $H$-types 7 through 10 we only note possible orbit lengths and the corresponding number of primitive designs $\operatorname{npd}_{H}(q)$ which we need for counting the total number of designs. Aut $D$ is easily read off from Table 1, except for $H$-type 8 where it is necessary to take into account Corollary 4.3 to obtain Aut $D$.

F) $H$-type $7\left(H \cong A_{5}\right)$

\begin{tabular}{|l|l|l|}
\hline \multicolumn{1}{|c|}{$q=p \equiv$} & $H=K \cong A_{5}$ orbit lengths & $\operatorname{npd}_{H}(q)$ \\
\hline \hline $1(\bmod 60)$ & $12^{1} 20^{1} 30^{1} 60^{\frac{q-61}{60}}$ & $2^{\frac{q+59}{60}}-1$ \\
\hline $11(\bmod 60)$ & $12^{1} 60^{\frac{q-11}{60}}$ & $2^{\frac{q-11}{60}}-1$ \\
\hline $19(\bmod 60)$ & $20^{1} 60^{\frac{q-19}{60}}$ & $2^{\frac{q-19}{60}}-1$ \\
\hline $29(\bmod 60)$ & $30^{1} 60^{\frac{q-29}{60}}$ & $2^{\frac{q-29}{60}}-1$ \\
\hline $31(\bmod 60)$ & $12^{1} 20^{1} 60^{\frac{q-31}{60}}$ & $2^{\frac{q+29}{60}}-1$ \\
\hline $41(\bmod 60)$ & $12^{1} 30^{1} 60^{\frac{q-41}{60}}$ & $2^{\frac{q+19}{60}}-1$ \\
\hline $49(\bmod 60)$ & $20^{1} 30^{1} 60^{\frac{q-49}{60}}$ & $2^{\frac{q+11}{60}}-1$ \\
\hline $59(\bmod 60)$ & $60^{\frac{q+1}{60}}$ & $2^{\frac{q-59}{60}}-1$ \\
\hline
\end{tabular}


G) $H$-type $8\left(H \cong A_{4}\right)$

\begin{tabular}{|l|l|l|l|}
\hline \multicolumn{1}{|c|}{$q=p \equiv$} & \multicolumn{1}{|c|}{$H \cong A_{4}$} & \multicolumn{1}{|c|}{$K \cong S_{4}$} & $\operatorname{npd}_{H}(q)$ \\
\hline \hline $53,77(\bmod 120)$ & $6^{1} 12^{\frac{q-5}{12}}$ & $6^{1} 24^{\frac{q-5}{24}}$ & $2^{\frac{q-17}{12}}+2^{\frac{q-29}{24}}-1$ \\
\hline $83,107(\bmod 120)$ & $12^{\frac{q+1}{12}}$ & $12^{1} 24^{\frac{q-11}{24}}$ & $2^{\frac{q-23}{12}}+2^{\frac{q-35}{24}}-1$ \\
\hline $13,37(\bmod 120)$ & $4^{2} 6^{1} 12^{\frac{q-13}{12}}$ & $6^{1} 8^{1} 24^{\frac{q-13}{24}}$ & $2^{\frac{q-1}{12}}+2^{\frac{q-13}{24}}-1$ \\
\hline $43,67(\bmod 120)$ & $4^{2} 12^{\frac{q-7}{12}}$ & $8^{1} 12^{1} 24^{\frac{q-19}{24}}$ & $2^{\frac{q-7}{12}}+2^{\frac{q-19}{24}}-1$ \\
\hline
\end{tabular}

н) $H$-type $9\left(H \cong S_{4}\right)$

\begin{tabular}{|l|l|l|}
\hline \multicolumn{1}{|c|}{$q=p \equiv$} & $H=K \cong S_{4}$ orbit lengths & $\operatorname{npd}_{H}(q)$ \\
\hline \hline $1,49,73,97(\bmod 120)$ & $6^{1} 8^{1} 12^{1} 24^{\frac{q-25}{24}}$ & $2^{\frac{q+23}{24}}-1$ \\
\hline $7,31,79,103(\bmod 120)$ & $8^{1} 24^{\frac{q-7}{24}}$ & $2^{\frac{q-7}{24}}-1$ \\
\hline $17,41,89,113(\bmod 120)$ & $6^{1} 12^{1} 24^{\frac{q-17}{24}}$ & $2^{\frac{q+7}{24}}-1$ \\
\hline $23,47,71,119(\bmod 120)$ & $24^{\frac{q+1}{24}}$ & $2^{\frac{q-23}{24}}-1$ \\
\hline
\end{tabular}

I) $H$-type $10\left(H \cong S_{4}\right)$

\begin{tabular}{|l|l|l|}
\hline$q=p \equiv$ & $H=K \cong S_{4}$ orbit lengths & $\operatorname{npd}_{H}(q)$ \\
\hline \hline $29,101(\bmod 120)$ & $6^{1} 24^{\frac{q-5}{24}}$ & $2^{\frac{q-5}{24}}-1$ \\
\hline $11,59(\bmod 120)$ & $12^{1} 24^{\frac{q-11}{24}}$ & $2^{\frac{q-11}{24}}-1$ \\
\hline $61,109(\bmod 120)$ & $6^{1} 8^{1} 24^{\frac{q-13}{24}}$ & $2^{\frac{q+11}{24}}-1$ \\
\hline $19,91(\bmod 120)$ & $8^{1} 12^{1} 24^{\frac{q-19}{24}}$ & $2^{\frac{q+5}{24}}-1$ \\
\hline
\end{tabular}

\section{SuRVEy OF RESUlts}

The $q$-range covered theoretically in this research is $q \geq 13, q \neq 23$. Cases with $q<13$ and $q=23$ are solved using programming and computation in GAP and MAGMA. In this way the nonexistence of primitive designs with $q=4,7,8,11$, and 23 is proved. For $q=23$ it is interesting that $\operatorname{PSL}(2,23)<M_{24}$ holds, [14], cf. Proposition 4.1. However, an exhausting computer search shows the nonexistence of primitive design with an automorphism group having $\operatorname{PSL}(2,23)$ as the socle.

Below we give the number of primitive designs obtained through exhaustive computer search for all $q \leq 103$. The designs and the related documentation are available at: http://www.pmfst.hr/ sbraic/t-designs/. 


\begin{tabular}{|c||c|c|c|c|c|c|c|c|c|c|c|c|c|}
\hline$q$ & 4 & 5 & 7 & 8 & 9 & 11 & 13 & 16 & 17 & 19 & 23 & 25 & 27 \\
\hline $\operatorname{npd}(q)$ & 0 & 1 & 0 & 0 & 2 & 0 & 4 & 1 & 3 & 1 & 0 & 3 & 2 \\
\hline
\end{tabular}

\begin{tabular}{|c||c|c|c|c|c|c|c|c|c|c|c|c|c|}
\hline$q$ & 29 & 31 & 32 & 37 & 41 & 43 & 47 & 49 & 53 & 59 & 61 & 64 & 67 \\
\hline $\operatorname{npd}(q)$ & 3 & 2 & 0 & 11 & 6 & 9 & 1 & 4 & 11 & 3 & 12 & 2 & 35 \\
\hline
\end{tabular}

\begin{tabular}{|c||c|c|c|c|c|c|c|c|c|}
\hline$q$ & 71 & 73 & 79 & 81 & 83 & 89 & 97 & 101 & 103 \\
\hline $\operatorname{npd}(q)$ & 4 & 17 & 8 & 3 & 35 & 18 & 33 & 20 & 15 \\
\hline
\end{tabular}

The sole existing design for $q=5$ has a block stabilizer $H$ of type 3 . It is a $2-(6,3,2)$ design to which extends the validity of Proposition 5.2. Out of two designs existing for $q=9$, one is $2-(10,4,2)$ design with a block stabilizer $H$ of type 4, described in Proposition 5.4, (2). The other is $3-(10,5,3)$ design with a block stabilizer $H=C_{5} \rtimes C_{4}$. The subgroup $H \cap T$ is not maximal in $T=\operatorname{PSL}(2,9)$, whereas $H$ is maximal in $M_{10}$, which is the full automorphism group of this design.

The total number of nontrivial primitive $t$-designs, up to isomorphism and complementation, for a given $q$ is the sum of $\operatorname{npd}(q)$ over all $H$-types. Due to the incompleteness of results for block stabilizers $H$ of type 5 , in the following proposition we give that number only for $q=p$. The proof is pure combinatorics.

Proposition 7.1. If $q \geq 7$ is prime then the following formulas hold:

1. $q \equiv 1(\bmod 120) \Rightarrow \operatorname{npd}(q)=2^{\frac{q+59}{60}}+2^{\frac{q+23}{24}}$,

2. $q \equiv 7,103(\bmod 120) \Rightarrow \operatorname{npd}(q)=2^{\frac{q-7}{24}}-1$,

3. $q \equiv 11(\bmod 120) \Rightarrow \operatorname{npd}(q)=2^{\frac{q-11}{60}}+2^{\frac{q-11}{24}}-2$,

4. $q \equiv 13,37(\bmod 120) \Rightarrow \operatorname{npd}(q)=2^{\frac{q-1}{12}}+2^{\frac{q-13}{24}}+1$,

5. $q \equiv 17,113(\bmod 120) \Rightarrow \operatorname{npd}(q)=2^{\frac{q+7}{24}}+1$,

6. $q \equiv 19(\bmod 120) \Rightarrow \operatorname{npd}(q)=2^{\frac{q-19}{60}}+2^{\frac{q+5}{24}}-2$,

7. $q \equiv 23,47(\bmod 120) \Rightarrow \operatorname{npd}(q)=2^{\frac{q-23}{24}}-1$,

8. $q \equiv 29(\bmod 120) \Rightarrow \operatorname{npd}(q)=2^{\frac{q-29}{60}}+2^{\frac{q-5}{24}}$,

9. $q \equiv 31(\bmod 120) \Rightarrow \operatorname{npd}(q)=2^{\frac{q+29}{60}}+2^{\frac{q-7}{24}}-2$,

10. $q \equiv 41(\bmod 120) \Rightarrow \operatorname{npd}(q)=2^{\frac{q+19}{60}}+2^{\frac{q+7}{24}}$,

11. $q \equiv 43,67(\bmod 120) \Rightarrow \operatorname{npd}(q)=2^{\frac{q-7}{12}}+2^{\frac{q-19}{24}}-1$,

12. $q \equiv 49(\bmod 120) \Rightarrow \operatorname{npd}(q)=2^{\frac{q+11}{60}}+2^{\frac{q+23}{24}}$,

13. $q \equiv 53,77(\bmod 120) \Rightarrow \operatorname{npd}(q)=2^{\frac{q-17}{12}}+2^{\frac{q-29}{24}}+1$,

14. $q \equiv 59(\bmod 120) \Rightarrow \operatorname{npd}(q)=2^{\frac{q-59}{60}}+2^{\frac{q-11}{24}}-2$,

15. $q \equiv 61(\bmod 120) \Rightarrow \operatorname{npd}(q)=2^{\frac{q+59}{60}}+2^{\frac{q+11}{24}}$,

16. $q \equiv 71(\bmod 120) \Rightarrow \operatorname{npd}(q)=2^{\frac{q-11}{60}}+2^{\frac{q-23}{24}}-2$,

17. $q \equiv 73,97(\bmod 120) \Rightarrow \operatorname{npd}(q)=2^{\frac{q+23}{24}}+1$,

18. $q \equiv 79(\bmod 120) \Rightarrow \operatorname{npd}(q)=2^{\frac{q-19}{60}}+2^{\frac{q-7}{24}}-2$, 
19. $q \equiv 83,107(\bmod 120) \Rightarrow \operatorname{npd}(q)=2^{\frac{q-23}{12}}+2^{\frac{q-35}{24}}-1$,

20. $q \equiv 89(\bmod 120) \Rightarrow \operatorname{npd}(q)=2^{\frac{q-29}{60}}+2^{\frac{q+7}{24}}$,

21. $q \equiv 91(\bmod 120) \Rightarrow \operatorname{npd}(q)=2^{\frac{q+29}{60}}+2^{\frac{q+5}{24}}-2$,

22. $q \equiv 101(\bmod 120) \Rightarrow \operatorname{npd}(q)=2^{\frac{q+19}{60}}+2^{\frac{q-5}{24}}$,

23. $q \equiv 109(\bmod 120) \Rightarrow \operatorname{npd}(q)=2^{\frac{q+11}{60}}+2^{\frac{q+11}{24}}$,

24. $q \equiv 119(\bmod 120) \Rightarrow \operatorname{npd}(q)=2^{\frac{q-59}{60}}+2^{\frac{q-23}{24}}-2$.

The number $\operatorname{npd}\left(p^{2}\right)$ is given in Proposition 6.2. Notice that $q=p^{f}, f>2$ can appear only for $H$-types $2,3,4$ and 5 .

Proposition 7.2. Let $\alpha, \beta$ be nonnegative integers. Then for the number of primitive designs with $q=p^{2^{\alpha} 3^{\beta}}$ we have the following:

1. $\operatorname{npd}\left(2^{2^{\alpha}}\right)=1, \alpha \geq 2$.

2. $\operatorname{npd}\left(2^{3^{\alpha}}\right)=1, \alpha \geq 2$.

3. $\operatorname{npd}\left(2^{2^{\alpha} 3^{\beta}}\right)=2, \alpha, \beta \geq 1$.

4. $\operatorname{npd}\left(p^{2^{\alpha}}\right)=3, p \neq 2$ and $\alpha \geq 2$.

5. $\operatorname{npd}\left(p^{3^{\alpha}}\right)=2, p \equiv 3(\bmod 4)$ and $\alpha \geq 1$.

6. $\operatorname{npd}\left(p^{3^{\alpha}}\right)=4, p \equiv 1(\bmod 4)$ and $\alpha \geq 1$.

7. $\operatorname{npd}\left(p^{2^{\alpha} 3^{\beta}}\right)=5, p \neq 2$ and $\alpha, \beta \geq 1$.

Proposition 7.3. Let $q \geq 4$. Then $\operatorname{npd}(q)=0$ if and only if $q=7,11,23$ or $q=2^{r}, r$ a prime.

Proof. If $q=7,11,23$ or $q=2^{r}, r$ a prime, we use Proposition 7.1, Proposition 5.1 and Proposition 5.2 to obtain $\operatorname{npd}(q)=0$. Conversely, let $\operatorname{npd}(q)=0$. If $q=p$, we simply solve the equalities $\operatorname{npd}(q)=0$ in Proposition 7.1. If $q=p^{f}, f \geq 2$, then there exists a prime $r \mid f$ so that $q=q_{0}^{r} \quad\left(q_{0}=p^{f / r}\right.$, this relates to $H$-types 4 and 5$)$ and it is known that 3 -designs $D\left(T,\{\infty\} \cup F_{q_{0}}\right)$ called spherical geometries exist, $[7$, p. 82]. The spherical geometry is not primitive design only in case $p=2$ and $f$ is a prime.

\section{REFERENCES}

[1] M. Aschbacher, Finite group theory, Cambridge University Press, Cambridge, 2000.

[2] T. Beth, D. Jungnickel and H. Lenz, Design theory, Cambridge University Press, 1999.

[3] W. Bosma, J. J. Cannon, C. Fieker, A. Steel (eds.), Handbook of Magma functions, Edition 2.16, 2010.

[4] P. J. Cameron, Permutation groups, Cambridge University Press, 1999.

[5] P. J. Cameron, H. R. Maimani, G. R. Omidi and B. Tayfeh-Rezaie, 3-designs from PSL(2, q), Discrete Math. 306 (2006), 3063-3073.

[6] P. J. Cameron, G. R. Omidi and B. Tayfeh-Rezaie, 3-designs from PGL(2, q), Electron. J. Combin. 13 (2006), Research Paper 50, 11 pp.

[7] C. J. Colbourn and J. H. Dinitz, Eds., Handbook of combinatorial designs, CRC Press, Boca Raton, 2007.

[8] J. D. Dixon and B. Mortimer, Permutation groups, Springer, New York, 1996. 
[9] The GAP Group, GAP - groups, algorithms, and programming, version 4.4; Aachen, St. Andrews, 2006 (http://www.gap--system.org).

[10] M. Giudici, Maximal subgroups of almost simple groups with socle $\operatorname{PSL}(2, q)$, arXiv:math/0703685v1 [math.GR], 2007.

[11] P. Kleidman and M. Liebeck, The subgroup structure of the finite classical groups, Cambridge University Press, Cambridge, 1990.

[12] R. Laue, Solving isomorphism problems for t-designs, in Designs 2002, 277-300, ed. W. D. Wallis, Kluwer Academic Publishers, Boston, 2003.

[13] M. W. Liebeck, C. E. Praeger and J. Saxl, On the O'Nan-Scott theorem for finite primitive permutation groups, J. Austral. Math. Soc. Ser. A 44 (1988), 389-396.

[14] M. W. Liebeck, C. E. Praeger and J. Saxl, A classification of the maximal subgroups of the finite alternating and symmetric groups, J. Algebra 111 (1987), 365-383.

[15] L. H. Soicher, The DESIGN package for GAP, Version 1.3, 2006, http:// designtheory.org/software/gap_design/.

\section{S. Braić}

Department of Mathematics

University of Split

Teslina 12/III, 21000 Split

Croatia

E-mail: sbraic@pmfst.hr

\section{J. Mandić}

Department of Mathematics

University of Split

Teslina 12/III, 21000 Split

Croatia

E-mail: majo@pmfst.hr

T. Vučičić

Department of Mathematics

University of Split

Teslina 12/III, 21000 Split

Croatia

E-mail: vucicic@pmfst.hr

Received: 29.1.2014. 